\title{
Estrategias de Innovación Desarrolladas por los Centros de Investigación de las Universidades Públicas del Departamento de la Guajira, Colombia*
}

\section{Innovation Strategies Developed by Research Centers in Public Universities of the Department of La Guajira, Colombia}

\author{
DOI: http://dx.doi.org/10.17981/ingecuc.12.1.2016.03
}

Artículo de Investigación Científica - Fecha de recepción: 20 de agosto de 2015 - Fecha de aceptación: 27 de noviembre de 2015

Lorena Esther Gómez Bermúdez

Doctor en Ciencia y Tecnología, Universidad de La Guajira. Riohacha (Colombia) lgomez@uniguajira.edu.co

\section{Edilberto Rafael Santos Moreno}

Magíster en Gerencia de Proyectos de Investigación, Universidad de La Guajira. Riohacha (Colombia) esantos@uniguajira.edu.co

\section{Martha Josefina Castrillón Rois}

Doctor en Ciencia y Tecnología, Universidad de La Guajira. Riohacha (Colombia) mcastrillon@uniguajira.edu.co

Para citar este artículo / To reference this paper:

L. E. Gómez Bermúdez, E. R. Santos Moreno y M. J. Castrillón Rois "Estrategias de Innovación Desarrolladas por los Centros de Investigación de las Universidades Públicas del Departamento de la Guajira, Colombia”, INGE CUC, vol. 12, no. 1, pp. 32-41, 2016. DOI: http://dx.doi.org/10.17981/ingecuc.12.1.2016.03

Resumen-- El propósito de este artículo es analizar las estrategias de innovación desarrolladas por los centros de investigación de las universidades públicas del Departamento de La Guajira, Colombia. La investigación es de tipo descriptivo, de diseño no experimental, transversal de campo. La población está conformada por la Universidad de La Guajira y la Universidad Nacional Abierta y a Distancia. La técnica de recolección de datos fue la encuesta con escala tipo Likert. Para el análisis de la información se determinó la media, interpretada a través de baremos con rango establecidos. Los resultados permitieron conocer que las universidades objeto de estudio no tienen estrategias de innovación diseñadas, ya que realizan pocas actividades de investigación y desarrollo, son pocos los incentivos otorgados a los investigadores, la cultura organizacional existente no fomenta la creatividad e innovación y se presenta un bajo uso de tecnologías de información y comunicación. Por lo anterior, es necesario establecer estrategias de innovación como herramientas esenciales para generar ventajas competitivas y alcanzar el éxito de estas universidades.

Palabras clave-- Cultura organizacional; factor humano; investigación; innovación; universidades públicas.

\begin{abstract}
The purpose of this article is to analyze the innovation strategies developed by the research centers of public universities in the department of La Guajira, Colombia. It is descriptive, not experimental, transversal design field; the population formed by the University of La Guajira and National Open University and Distance. The data collection technique was the survey with Likert For information analysis determined the average, interpreted through scales with established range scale. The results point to the universities under study have designed strategies of innovation, as they perform a few research and development, there are few incentives given to researchers, the existing organizational culture does not encourage creativity and innovation, it has a low use of information and communication technologies. Therefore, it is necessary to establish innovation strategies as essential tool to generate competitive advantages and success of these universities.
\end{abstract}

Keywords-- Organizational Culture, Human Factor; Research; Innovation; Public Universities.

\footnotetext{
"Artículo de Investigación Científica derivado del proyecto de investigación titulado "Innovación como Política Pública en los Centros de Investigación de las Instituciones Públicas de Educación Superior del Departamento de La Guajira." Financiado por el Centro de Investigación de la Universidad de La Guajira, Fecha inicio: febrero de 2013 - Fecha finalización: enero de 2015.
} 


\section{INTRODUCCIÓN}

En este mercado globalizado de hoy, donde se emplea el conocimiento como factor clave para generar valor, se adoptan como eje principal la investigación y desarrollo como fuentes de innovación para alcanzar la competitividad y crecimiento sostenido de las organizaciones. Específicamente, las instituciones de educación superior deben renovar continuamente los procesos de docencia, investigación y proyección social para lograr un liderazgo y posicionamiento a nivel nacional e internacional.

Se resalta que cada día se presentan evidencias de la relación que existe entre el desarrollo tecnológico y el crecimiento económico de las regiones y de los países; desde esa perspectiva, se reconoce que la inversión en ciencia, tecnología e innovación ha sido una de las fuentes más importantes de la transformación económica y tecnológica que han contribuido a dinamizar y acelerar la transformación productiva del país [1].

Las instituciones de educación superior, para cumplir con el proceso de docencia, investigación y proyección, deben jugar un papel fundamental como organizaciones innovadoras en la producción y transferencia de conocimientos así como de tecnología, donde la innovación constituya el eje de una nueva cultura académica; sin embargo, en la investigación titulada Innovación y productividad científica en los institutos universitarios de tecnología de la región andina de Venezuela, realizada por [2], pone en evidencia la ausencia de políticas y estrategias de innovación que refuercen su compromiso social, calidad, pertinencia y responsabilidad como factores impulsores del éxito.

Se considera que en las universidades no existen políticas internas que impulsen el desarrollo de la Ciencia, Tecnología e Innovación (CTI), tampoco se han diseñado mecanismos eficientes de transferencia de conocimiento generado del quehacer investigativo, por lo cual existe una escasa difusión de resultados de investigación que permita generar nuevos conocimientos sobre la base de los ya existentes; asimismo, se presenta duplicidad de los trabajos de investigación, ya que no existe una base de datos permanentemente actualizada. También es poca la información que tiene el sector productivo acerca de la función y de lo que producen los centros de investigación de las instituciones de educación superior; ese conocimiento, permitiría a las universidades conocer las necesidades del sector empresarial y productivo [3].

Concretamente, las universidades públicas del Departamento de La Guajira están sometidas a una crisis presupuestaria que se evidencia en retrasos en los depósitos de las asignaciones monetarias por parte de la administración departamental y nacional, lo que implica la contracción de los recursos económicos dirigidos a desarrollar $\mathrm{I}+\mathrm{D}+\mathrm{I}$, impidiendo además, la posibilidad de otorgar estímulos a los investigadores pertenecientes a grupos avalados por las universidades y categorizados por COLCIENCIAS.

Además, en estas instituciones universitarias no se han diseñado políticas internas de financiación de las actividades de I+D+I y tienen una débil infraestructura tecnológica para el desarrollo de este tipo de actividades debido a que no se tienen establecidas estrategias de innovación e investigación.

Se sustenta entonces la intención de realizar una investigación cuyo objetivo sea analizar las estrategias de innovación utilizadas por los centros de investigación constituidos dentro de las instituciones públicas de educación superior del Departamento de La Guajira, Colombia, sobre la base de las ideas expuestas y atendiendo la política educativa del gobierno colombiano, cuyo objetivo es transformar el sistema educativo en magnitud y pertinencia para garantizar la competitividad del país, conseguir una mejor calidad de vida y mayor equidad, teniendo claro que esta transformación se logra a través de las universidades, las cuales de manera permanente deben constituir alianzas estratégicas estado-universidad-empresa.

Se espera que los resultados de este estudio sean de gran utilidad a las instituciones del sector para implementar planes de mejoramiento en sus procesos de investigación y proyección social a fin de incrementar su eficiencia, eficacia y efectividad, así como de asegurar su proceso de desarrollo científico-tecnológico.

\section{ReVisión Literaria}

\section{A. Estrategias de innovación}

Una de las funciones misionales e importantes en las universidades es la investigación, ya que por medio de ella se genera nuevo conocimiento y se busca la aplicabilidad de ese conocimiento a la solución de los problemas del entorno social.

La investigación que contribuya a resolver problemas de índole político, económico y social, un recurso humano altamente calificado y la implementación de nuevos laboratorios y equipo tecnológico son fundamentales para la competitividad de las universidades.

La referencia [4] sostiene que una universidad, para que sea considerada como innovadora, debe cumplir con algunas condiciones (personal con competencias para realizar I+D, infraestructura, capacidad instalada, entre otros) que hagan énfasis en las características propias de la institución, en las actividades que se llevan a cabo dentro de ella, en la manera cómo se financia y los resultados que se obtienen a través de sus grupos de investigación.

En las instituciones con experiencia en gestión científica y tecnológica de investigación universi- 
taria, los esfuerzos deben encaminarse a diseñar y proponer una estrategia que permita incrementar la actividad científica; el reto lo constituye organizar la actividad mediante una estructura que permita poner en marcha esa estrategia, de tal forma que los profesores puedan desarrollar sus actividades de docencia y de igual forma preparar, presentar y ejecutar proyectos de investigación con recursos de financiación nacionales e internacionales que permitan resultados científicos y tecnológicos que impacten a la sociedad [5].

Por lo anterior, es necesario que en las universidades se establezcan estrategias de innovación para generar ventajas competitivas y alcanzar su máximo desarrollo. Estrategia que se puede definir en términos de lo que puede hacer una institución educativa; es decir, sus fortalezas y debilidades, así como las posibilidades que tiene como organización generadora de conocimiento. De la misma manera, la referencia [2] sostiene que la estrategia es un plan que se propone dotar a la institución de una ventaja competitiva sobre las instituciones del mismo sector a través de la diferenciación.

Desde esa perspectiva, se puede afirmar que las estrategias son acciones potenciales que requieren decisiones de la alta dirección y recursos por parte de las instituciones universitarias para conseguir los objetivos establecidos, es decir, ordenar los recursos para ser más eficientes así como más competitivas [6].

El mismo autor afirma, que se deben diseñar estrategias de innovación para incrementar tanto la competitividad como la rentabilidad de las instituciones de educación superior mediante el fomento de la innovación y la mejora continua de la calidad de los servicios suministrados a los usuarios. La innovación es considerada como una actividad que crea tanto valor social como económico, poniendo en juego un proceso de aprendizaje colectivo en el que se intercambia y se crea conocimiento entre las personas de la organización y de su entorno [7].

Desde ese aspecto, existe una serie de factores internos y externos implícitos en la determinación de la misión y objetivos que afectan la formulación de una estrategia de innovación en cualquier nivel de las instituciones universitarias [6]. En primer lugar, la estrategia debe tratar de aprovechar las oportunidades y neutralizar las amenazas del entorno analizando las fuerzas externas que influyen potencialmente en la institución.

En segunda instancia, se debe sopesar la totalidad de recursos disponibles y valorar de manera objetiva las fortalezas y debilidades en relación con las demás instituciones del mismo sector.

En tercer lugar, se debe tomar en cuenta la historia de las instituciones y su forma de responder ante los compromisos adquiridos; toda comprensión del futuro se origina en la reflexión y en una mirada al pasado.
En cuarto lugar, las ambiciones, las actitudes hacia el riesgo y los principios éticos de los directivos ejercen una fuerte influencia en la determinación de la estrategia de innovación.

En quinto lugar, para la formulación de la estrategia se consideran los grupos de interés, stakeholders, es decir, aquellos capaces de afectar o ser afectados por los objetivos, estrategias y prácticas, que en mayor o menor grado deben ser satisfechos. Estos grupos, internos o externos a la institución, están constituidos por los docentes, administrativos, directivos, estudiantes, proveedores, gobierno, sindicatos, comunidades locales, organizaciones no gubernamentales, entre otros.

Finalmente, en sexto lugar, la estrategia es afectada por la cultura organizacional, creencias y supuestos básicos compartidos por los miembros de la institución universitaria. Este factor puede convertirse en un propiciador básico del impulso innovador y potenciar la estrategia o, por el contrario, entorpecer su funcionamiento; en consecuencia, la cultura estimula la producción de resultados.

Una estrategia correctamente formulada, tomando en cuenta los seis aspectos descritos anteriormente, e implementada de manera eficaz y eficiente, proporcionará beneficios elevados con el consiguiente valor agregado, satisfacción de los estamentos universitarios y respaldo a la labor de los directivos.

Por otro lado, se considera que para desarrollar con éxito la estrategia de innovación es necesario determinar las capacidades disponibles en la institución con sus posibilidades de ampliación, seleccionar y planificar el accionar en el proceso de investigación, docencia y proyección social. Se trata de un conjunto de estrategias relacionadas entre sí; en primera instancia, una estrategia basada en conocimientos y capacidades para mejorar el logro de los objetivos de las instituciones [7].

Desde esa perspectiva, los centros de investigación de las instituciones de educación superior deben diseñar estrategias que generen planes de innovación que promuevan la producción y generación de conocimiento a través de la ejecución de proyectos de investigación, desarrollo tecnológico e innovación. Para lograr esto, es fundamental contar con un equipo humano con alto nivel de formación dedicado a la investigación científica y tecnológica que trabaje en red, abriendo camino para el logro de un desarrollo sostenible acorde con la biodiversidad del país y buscando aprovechar las oportunidades económicas y socio-culturales que presenta día a día el mundo globalizado.

Teniendo en cuenta los anteriores planteamientos, las estrategias pueden considerarse como acciones potenciales que requieren decisiones de la alta dirección y la asignación de recursos por parte de las organizaciones, entre estas las instituciones de educación superior y específicamente los centros 
de investigación, lo que permitirá cumplir con sus funciones y alcanzar los objetivos propuestos.

En función de lo expuesto, resulta acertado vincular la estrategia de innovación en las instituciones de educación universitaria considerando como dimensiones: el desarrollo de actividades de investigación y desarrollo (I+D), las habilidades y capacidades del capital humano, la cultura organizacional y las tecnologías de información y comunicación (TIC), elementos que permiten alcanzar el logro de los objetivos trazados y crear valor social ampliando los límites del conocimiento en los centros de investigación de las instituciones de educación superior.

\section{B. Desarrollo de actividades de investigación y desarrollo $(I+D)$}

La realización de actividades internas de $\mathrm{I}+\mathrm{D}$ ha sido una de las estrategias clásicas de innovación y representa un mecanismo por excelencia para organizar y dirigir los procesos innovadores en las instituciones. Según el autor, el desarrollo de actividades internas de $\mathrm{I}+\mathrm{D}$, como estrategia de innovación, ha sido un tema ampliamente abordado en la literatura [8].

De hecho, la totalidad de la investigación y desarrollo se considera como una actividad de innovación e incluye los trabajos de creación emprendidos con el fin de incrementar la suma de conocimientos, así como su utilización para concebir nuevas aplicaciones [9]. Estos patrones han evolucionado desde el establecimiento de grandes laboratorios corporativos de $\mathrm{I}+\mathrm{D}$, hasta la externalización de dichas actividades a través de la subcontratación con otras instituciones.

En esta línea uno de los aspectos más estudiados han sido los factores que determinan la decisión de las organizaciones de llevar a cabo actividades de I+D, y más concretamente, los elementos que influyen en el nivel de inversión en dichas actividades. En ese sentido, las universidades exclusivas de investigación y desarrollo, cuentan con recursos humanos de alto nivel y con una alta dedicación a investigación y desarrollo que compilan la investigación en laboratorios, institutos, centros y programas de investigación y escuelas de doctorado [5].

Según [10], la I+D puede abarcar distintos tipos de actividades:

- Investigación básica o fundamental, es decir, trabajos teóricos o empíricos para obtener un mayor grado de conocimiento de los fundamentos de los fenómenos y hechos observables, pero sin aplicación práctica inmediata.

- Investigación aplicada, la cual consiste en trabajos originales para adquirir un grado de conocimiento pero tiene siempre una misión práctica, aunque sea a largo plazo.
- Desarrollo experimental, el cual se trata de trabajos sistemáticos de profundización en los conocimientos existentes derivados de la investigación o la experiencia práctica dirigidos a la producción de nuevos materiales o productos, al establecimiento de nuevos productos o servicios, o a la mejora sustancial de los ya producidos o implantados.

Lo anterior, teniendo en cuenta que la función de la investigación científica no consiste únicamente en crear conocimientos científicos sino en aplicarlos en beneficio de toda la sociedad. En ese sentido, el rol de la educación superior es esencial para crear la capacidad intelectual de producir y utilizar conocimientos para el aprendizaje permanente, para actualizar conocimientos y habilidades que conlleven a la generación de innovaciones, cuestiones de importancia en una sociedad en la que el conocimiento es el principal motor de desarrollo y crecimiento económico. La ciencia debe cumplir con esa función social, los investigadores tienen el compromiso de poner la evidencia científica al servicio de todos los seres humanos [11].

Lo anteriormente señalado, permite apreciar las razones por las cuales resulta importante analizar el desarrollo de actividades de I+D como dimensión de las estrategias de innovación en el contexto de las universidades.

\section{Capital humano}

El capital humano es un creador de valor económico a través de su proceso intelectual y de los conocimientos adquiridos y capitalizados en alguna actividad productiva [12]. Por otro lado, el capital humano se puede considerar como la combinación de competencia y compromiso de los empleados de la organización para llevar a cabo la tarea que recae bajo su responsabilidad. Establece una clara relación entre la competencia y el conocimiento tácito y explícito, mientras que el compromiso se asocia con la motivación de los trabajadores para llevar a cabo las actividades que le fueron asignadas [6].

El capital humano constituye uno de los factores determinantes para la obtención de valor agregado en las organizaciones. Este valor se potencia cuando el conocimiento se coloca en función del logro de los objetivos de la organización. El capital humano depende en gran medida de la capacidad de las organizaciones para desarrollar y aprovechar el conocimiento [13].

Si la organización cuenta con un capital humano suficientemente calificado para llevar a cabo proyectos de innovación, entonces decidirá generar internamente la innovación [14]. Del capital humano parte el conocimiento, las habilidades, los valores y el potencial innovador de la organización, entre otros elementos. La gestión de dicho capital requiere de una atención muy especial que supone la capacidad 
de los directivos de identificar, medir, desarrollar y renovar el activo intangible para el futuro éxito de la organización. De ahí que el desarrollo económico de una organización, depende en gran medida de la creatividad de su capital humano para imaginar cosas nuevas, de su habilidad para buscar una aplicación práctica a esas cosas nuevas y de su capacidad para dar a conocer esas cosas nuevas en el mercado. Es importante que previo a tomar decisiones clave como lo es desarrollar innovaciones en la organización, se considere si se cuenta con capital humano suficientemente calificado para llevar a cabo proyectos de innovación [15].

Los planteamientos anteriores, dejan entrever la necesidad de que las organizaciones, tanto públicas como privadas, inviertan en la formación del recurso humano para alcanzar la eficiencia y eficacia de sus empleados en el logro de la mayor productividad y competitividad. Las instituciones de educación superior, como entes generadores de conocimiento deben invertir recursos en la formación de alto nivel de sus docentes e investigadores para que puedan adquirir las habilidades y competencias necesarias para desarrollar innovaciones en sus funciones, incrementando así la competitividad de estas organizaciones.

Lo anteriormente señalado, permite apreciar la importancia y la necesidad que tienen las universidades de contar con un capital humano con competencias para la realización de actividades de I+D que propicie la generación de innovación en los procesos de investigación, docencia y proyección social.

\section{Cultura organizacional}

Las instituciones están compuestas de personas que viven en ambientes complejos y dinámicos, lo que genera comportamientos diversos que influyen en el funcionamiento de los sistemas, y que se organizan en grupos y colectividades. El resultado de esta interacción media en el ambiente que se respira en la organización, entre ellas las instituciones de educación superior, de ahí la importancia de analizar el término cultura organizacional y tomarlo como un importante elemento que determina la forma de interacción de los miembros de una institución [16].

La cultura organizacional es el conjunto de suposiciones, creencias, valores y normas que comparten los miembros de las instituciones [17]. Otros autores consideran la cultura organizacional como una forma de vida, un conjunto de creencias y valores, una manera aceptada de interactuar y relacionarse en una determinada organización [18]. También se refiere a un sistema de significados compartidos entre sus miembros [19].

Los mencionados autores consideran que la cultura reviste importancia cuando está relacionada con la innovación. Sostener un ambiente que fomente la creatividad de nuevas e innovadoras ideas no es una tarea fácil, se requiere apoyar las actividades de innovación en los elementos organizacionales filosóficos, tales como los valores, las creencias, las motivaciones, el trabajo en equipo, la estructura de las organizaciones, las funciones y las normas de la institución. Desde una visión más amplia, la cultura no sólo incluye valores, actitudes y comportamientos, sino también las estrategias y las acciones que en conjunto funcionan como un sistema dinámico [20].

Por lo anterior, las instituciones de educación superior, para desarrollar innovaciones y ser competitivas, sólo tienen dos caminos: el primero es asumir la innovación, a pesar de los riesgos que se toman, y segundo, hacerla parte de su cultura, de su forma de ser. Para facilitar los procesos de innovación, se requiere que exista una cultura innovadora que genere un clima organizacional que estimule la creatividad en los empleados, pero para ello se necesita el esfuerzo de todos los trabajadores dentro de las instituciones [21]. Del análisis de diferentes definiciones se puede inferir que los distintos autores coinciden en afirmar que la cultura tiene elementos afines que comparten e identifican a una institución, haciendo que en sus miembros se fomente la creatividad e innovación y se profesen las mismas normas, valores, filosofías, reglas, lenguaje, ideologías, actitudes, mitos y ceremonias, entre otros.

Cabe de anotar que las instituciones de educación superior, como organizaciones formales, tienen un sistema de tareas bien definidas, cada una de las cuales lleva en sí misma una cantidad específica de autoridad y responsabilidad por parte de los actores, y estos tienen un determinado comportamiento, se asocian en agrupaciones particulares, respetan líneas de autoridad y establecen canales de comunicación entre sus miembros; son instituciones en las cuales la cultura organizacional es uno de los pilares fundamentales para el logro de los objetivos de cada facultad y programa de la institución. Esto se convierte en un motivo para incluirla como una importante dimensión para analizar las estrategias de innovación.

\section{E. Tecnologías de Información y Comunicaciones (TIC)}

La incorporación de las Tecnologías de Información y Comunicación (TIC) en el mundo ha suscitado un proceso de unificación en la comunicación sin precedentes que ha afectado a las actividades y saberes de la humanidad. Por medio de ellas se han creado espacios virtuales que propician que las personas intercambien, interactúen y generen conocimientos. Es a partir de esta tendencia que surge la relevancia de analizar las formas como se está llevando a cabo el uso de estas tecnologías en todos los ámbitos, pero particularmente en el medio universitario [22].

El Estado colombiano, mediante la Ley 1341 de 2009 , reconoce que el acceso y uso de las TIC, el despliegue, uso eficiente de la infraestructura, el desa- 
rrollo de aplicaciones, la protección a los usuarios, la formación de talento humano en estas tecnologías y su carácter transversal son pilares para la consolidación de las sociedades de la información. Se reconoce que la universidad como una organización de conocimiento debe dinamizar sus procesos mediante la incorporación y uso de las TIC [23].

En la gestión de la investigación, las TIC facilitan, de una parte, el trabajo colaborativo a través de la comunicación sincrónica o asincrónica entre los miembros de un grupo de investigación o entre grupos a nivel nacional o internacional; por otro lado, facilitan la comunicación de los resultados de investigación y su accesibilidad a la comunidad en general y a las comunidades científicas en particular, lo cual puede hacerse a través de la publicación de los resultados de investigación [24].

Para hacer buen uso de estas importantes herramientas tecnológicas, las nuevas tecnologías no deben ser calificadas sólo como meros instrumentos o medios, deben ser consideradas más bien como un nuevo ambiente que incluye todo dentro de sí. Se constituyen en un soporte fundamental de las estrategias competitivas y de la modernización de las organizaciones del sector universitario [25]. Teniendo en cuenta que las TIC se consideran como uno de los pilares básicos de las organizaciones y la sociedad en general, es importante considerarla como una de las estrategias de innovación en los centros de investigación de las instituciones de educación superior.

\section{III.MetodologíA}

Para el desarrollo de esta investigación, la variable estrategias de innovación se opera a través de las dimensiones: Desarrollo de actividades de I+D, Capital humano, Cultura organizacional y Tecnologías de Información y Comunicación (Tabla I).

Tabla I. Cuadro de Operación de la Variable

\begin{tabular}{|c|c|c|}
\hline Variable & Dimensiones & Indicadores \\
\hline \multirow{10}{*}{ 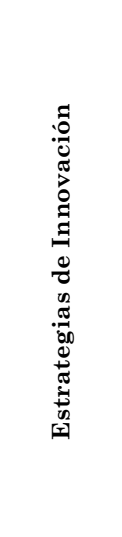 } & \multirow{3}{*}{$\begin{array}{l}\text { Desarrollo de } \\
\text { actividades de } \\
\text { I+D }\end{array}$} & Personal disponible \\
\hline & & Presupuesto \\
\hline & & Incentivos \\
\hline & \multirow{2}{*}{$\begin{array}{l}\text { Capital } \\
\text { humano }\end{array}$} & Conocimiento \\
\hline & & Estímulos a investigadores \\
\hline & \multirow{3}{*}{$\begin{array}{l}\text { Cultura } \\
\text { organizacional }\end{array}$} & Valores organizacionales \\
\hline & & Trabajo en equipos \\
\hline & & Clima organizacional \\
\hline & \multirow{2}{*}{$\begin{array}{l}\text { Tecnologías de } \\
\text { Información y } \\
\text { Comunicación }\end{array}$} & $\begin{array}{l}\text { Uso para redes de } \\
\text { conocimiento }\end{array}$ \\
\hline & & $\begin{array}{l}\text { Herramienta para actividades } \\
\text { de innovación }\end{array}$ \\
\hline
\end{tabular}

Fuente: Autor
El tipo de estudio fue descriptivo de diseño no experimental, transeccional y de campo. La población está conformada por los centros de investigación de las instituciones públicas de educación superior del Departamento de La Guajira, Colombia: Universidad de La Guajira y Universidad Nacional Abierta y a Distancia, tomando como unidades de información a los vicerrectores de investigación, los directores de investigación y las personas con características de líderes de los grupos de investigación, para un total de 45 personas.

El instrumento utilizado para la recolección de datos fue un cuestionario con escala de Likert, el cual fue diseñado dividiendo cada pregunta en cinco (5) posibles alternativas de repuesta, como son: Totalmente de acuerdo (TA), Medianamente de acuerdo (MA), Ni de acuerdo ni en desacuerdo (NAND), Medianamente en desacuerdo (MD), Totalmente en desacuerdo (TD), a las cuales se les asignó una calificación del 1 al 5 para una mejor tabulación de las respuestas, tal como se aprecia en la Tabla II.

TABla II. VAlor establecido Para CADA OPCIÓN DE LA EscALA LIKERT

\begin{tabular}{|l|c|c|}
\hline \multicolumn{1}{|c|}{ Escala de Likert } & Opción & Valor \\
\hline Totalmente de acuerdo & TA & 5 \\
\hline Medianamente de acuerdo & MA & 4 \\
\hline Ni de acuerdo ni en desacuerdo & NAND & 3 \\
\hline Medianamente en desacuerdo & MD & 2 \\
\hline Totalmente en desacuerdo & TD & 1 \\
\hline
\end{tabular}

Fuente: Autor

Para el análisis de cada una de las dimensiones e indicadores se decidió utilizar los parámetros establecidos en la estadística descriptiva, en la cual se emplea la media como medida de tendencia central. Para la interpretación de los estadísticos descriptivos y elaboración de las tablas de resultados, así como para la interpretación de la media, fueron utilizados baremos, como se presenta en la Tabla III.

Tabla III. AnÁlisis de la Media

\begin{tabular}{|c|c|l|}
\hline \multicolumn{3}{|c|}{ Media } \\
\hline Rango & Intervalo & \multicolumn{1}{c|}{ Categoría } \\
\hline 5 & $4,20<\bar{x} \leq 5.00$ & Muy alto nivel \\
\hline 4 & $3,4<\bar{x} \leq 4.20$ & Alto nivel \\
\hline 3 & $2,60<\bar{x} \leq 3,40$ & Moderado nivel \\
\hline 2 & $1.80<\bar{x} \leq 2.60$ & Bajo nivel \\
\hline 1 & $1,00 \leq \bar{x} \leq 1,80$ & Muy bajo nivel \\
\hline
\end{tabular}

Fuente: Autor. 


\section{Resultados}

A continuación se presentan los resultados obtenidos al analizar las estrategias de innovación desarrolladas en los centros de investigación de las universidades objeto de estudio.

\section{A. Dimensión: Desarrollo de actividades de $I+D$}

En la Tabla IV se presentan los resultados obtenidos para la dimensión Desarrollo de Actividades de $I+D$, específicamente en el indicador Personal disponible los resultados demuestran que el $67 \%$ de las unidades de información expresaron que están medianamente en desacuerdo sobre el hecho de que haya disponibilidad de personal con las competencias necesarias para el desarrollo de actividades de I+D; el $15 \%$ está ni de acuerdo ni en desacuerdo, el $9 \%$ está totalmente de acuerdo, el 7\% está totalmente en desacuerdo y el $2 \%$ está medianamente de acuerdo.

Haciendo referencia a la media se observa que ésta arrojó un resultado de 2.4, ubicándola en una categoría de bajo nivel de acuerdo con el baremo de medición previamente establecido; esto indica que hay un bajo nivel de personal disponible con las competencias necesarias para el desarrollo de actividades de I+D.

Tomando en consideración los resultados del indicador Presupuesto se observa que la alternativa con mayor número de respuestas es Medianamente en desacuerdo con un $56 \%$, seguida de $\mathrm{Ni}$ de acuerdo ni en desacuerdo con un $18 \%$, lo cual indica que de manera baja se destina presupuesto para las actividades de I+D. En cuanto a la media, se observa un valor de 2.42 , ubicándola en la categoría de bajo nivel según el baremo de medición previamente establecido.
Respecto al indicador Incentivos, se puede observar que la alternativa con mayor número de respuestas es Medianamente en desacuerdo con un $44 \%$, seguida de Ni de acuerdo ni en desacuerdo con un $16 \%$ y Totalmente en desacuerdo con un $15 \%$, mostrando que de manera baja se incentiva al personal para el desarrollo de actividades de I+D. En cuanto a la media, se puede ver que refleja un valor de 2.68, ubicándola en la categoría de moderado nivel según el baremo de medición previamente establecido. Los resultados anteriormente descritos indican un valor de la media para la dimensión Desarrollo de actividades de $I+D$ de 2.5 , lo que refleja un bajo nivel en el desarrollo de este tipo de actividades en los centros de investigación de las universidades objeto de estudio.

\section{B. Dimensión: Capital humano}

En la Tabla V, se presentan los resultados obtenidos para la dimensión Capital humano; con referencia al indicador Conocimiento, el 35\% de los sujetos encuestados indicaron que están medianamente en desacuerdo en que se aprovechan los conocimientos del talento humano, mientras que el 33\% expresó estar totalmente de acuerdo y el $16 \%$ manifestó estar medianamente de acuerdo. En cuanto a la media, los resultados muestran un valor de 3.15, correspondiente a la categoría de moderado nivel según el baremo de medición previamente establecido.

En relación con el indicador Estímulos a investigadores, el 33\% de los sujetos encuestados indicaron que están medianamente de acuerdo en que se otorga estímulos a los investigadores por su aporte a la CTeI, el $29 \%$ está totalmente de acuerdo y el $22 \%$ esta medianamente en desacuerdo.

Tabla IV. Estadísticos para la Dimensión Desarkollo de Actividades de I+D

\begin{tabular}{|c|c|c|c|c|c|c|c|c|c|c|c|}
\hline \multirow{2}{*}{ Indicadores } & \multicolumn{2}{|c|}{ TA } & \multicolumn{2}{|c|}{ MA } & \multicolumn{2}{|c|}{ NAND } & \multicolumn{2}{|c|}{ MD } & \multicolumn{2}{|c|}{ TD } & \multirow[t]{2}{*}{ Media } \\
\hline & $\mathbf{F a}$ & Fr & $\mathbf{F a}$ & Fr & $\mathbf{F a}$ & Fr & $\mathbf{F a}$ & Fr & $\mathbf{F a}$ & Fr & \\
\hline Personal disponible & 4 & $9 \%$ & 1 & $2 \%$ & 7 & $15 \%$ & 30 & $67 \%$ & 3 & $7 \%$ & 2.4 \\
\hline Presupuesto & 2 & $5 \%$ & 5 & $11 \%$ & 8 & $18 \%$ & 25 & $56 \%$ & 5 & $11 \%$ & 2.42 \\
\hline Incentivos & 6 & $13 \%$ & 5 & $11 \%$ & 7 & $16 \%$ & 20 & $44 \%$ & 7 & $15 \%$ & 2.68 \\
\hline \multicolumn{11}{|c|}{ Total } & 2.5 \\
\hline
\end{tabular}

Fuente: Autor.

Tabla V. Estadísticos para la Dimensión Capital Humano

\begin{tabular}{|c|c|c|c|c|c|c|c|c|c|c|c|}
\hline \multirow{2}{*}{ Indicadores } & \multicolumn{2}{|c|}{ TA } & \multicolumn{2}{|c|}{ MA } & \multicolumn{2}{|c|}{ NAND } & \multicolumn{2}{|c|}{ MD } & \multicolumn{2}{|c|}{ TD } & \multirow{2}{*}{ Medía } \\
\hline & $\mathbf{F a}$ & Fr & $\mathbf{F a}$ & FR & $\mathbf{F a}$ & Fr & FA & FR & $\mathbf{F a}$ & Fr & \\
\hline Conocimiento & 15 & $33 \%$ & 7 & $16 \%$ & 3 & $7 \%$ & 16 & $35 \%$ & 4 & $9 \%$ & 3.28 \\
\hline Estímulos a investigadores & 13 & $29 \%$ & 15 & $33 \%$ & 0 & $\%$ & 10 & $22 \%$ & 7 & $16 \%$ & 3.37 \\
\hline \multicolumn{11}{|c|}{ Total } & 3.33 \\
\hline
\end{tabular}

Fuente: Autor 
En cuanto a la media, los resultados demuestran un valor de 3.37, correspondiente a la categoría de moderado nivel según el baremo de medición previamente establecido. Los resultados descritos muestran que la dimensión Capital humano obtuvo un valor de la media de 3.33 , lo que indica un moderado nivel, según el baremo establecido, reflejando que de manera moderada se tiene en cuenta la importancia del talento humano para generar innovaciones en los procesos desarrollados por los centros de investigación de las universidades objeto de estudio.

\section{Dimensión: Cultura organizacional}

En la Tabla VI, se presentan los resultados obtenidos de la dimensión Cultura organizacional. Se observa que para el indicador Valores organizacionales el $67 \%$ de los sujetos encuestados indicaron estar medianamente en desacuerdo en que se promueve la internalización de los valores organizacionales entre sus miembros, mientras que el $29 \%$ indicó estar ni de acuerdo ni en desacuerdo y el $4 \%$ manifiesta estar totalmente en desacuerdo. En cuanto a la media, los resultados muestran un valor de 2.24, correspondiente a la categoría de bajo nivel según el baremo de medición previamente establecido.

En referencia al indicador Trabajo en equipo se puede observar que el $56 \%$ de los encuestados está de acuerdo en que se promueve el trabajo en equipo de los investigadores, el 40\% está medianamente de acuerdo y sólo el $4 \%$ está totalmente de acuerdo. La media de este indicador obtuvo un valor de 2.93, ubicándose en la categoría de moderado nivel según el baremo de medición diseñado.

\section{Dimensión: Tecnologías de Información y Comunicación}

En la Tabla VII, se presentan los resultados obtenidos para la dimensión Tecnologías de Información y Comunicación; específicamente, para el indicador Uso para redes de conocimiento el $64 \%$ de los sujetos encuestados indicaron estar medianamente en desacuerdo en que se promueve el uso de las TIC para realizar contactos con redes de conocimiento, mientras que el $31 \%$ indicó estar ni de acuerdo ni en desacuerdo y el $4 \%$ manifiesta estar totalmente en desacuerdo. En cuanto a la media, los resultados muestran un valor de 2.3, correspondiente a la categoría de bajo nivel según el baremo de medición previamente establecido.

Con respecto al indicador Herramienta para actividades de innovación se muestra que el 56\% de los sujetos encuestados manifestaron estar medianamente en desacuerdo en que se promueve el uso de las TIC como herramientas para el desarrollo de actividades de innovación. Para este indicador se obtuvo un valor de la media de 2.8, reflejándose un moderado nivel según el baremo de medición previamente establecido.

Los resultados obtenidos en la dimensión Tecnologías de Información y Comunicación, muestran que tiene un valor de la media de 2.5 , lo que indica un bajo nivel, según el baremo establecido. Esto refleja que de manera baja se promueve el uso de las TIC como herramienta fundamental para establecer estrategias de innovación en los centros de investigación de las universidades objeto de estudio.

Tabla VI. Estadísticos para la Dimensión Cultura Organizacional

\begin{tabular}{|c|c|c|c|c|c|c|c|c|c|c|c|}
\hline \multirow{2}{*}{ Indicadores } & \multicolumn{2}{|c|}{ TA } & \multicolumn{2}{|c|}{ MA } & \multicolumn{2}{|c|}{ NAND } & \multicolumn{2}{|c|}{ MD } & \multicolumn{2}{|c|}{ TD } & \multirow[t]{2}{*}{ Media } \\
\hline & fa & fr & fa & fr & $\mathbf{F a}$ & fr & $\mathbf{F a}$ & $\mathbf{F r}$ & fa & fr & \\
\hline Valores organizacionales & 0 & $0 \%$ & 0 & $0 \%$ & 13 & $29 \%$ & 30 & $67 \%$ & 2 & $4 \%$ & 2.24 \\
\hline Trabajo en equipo & 2 & $4 \%$ & 18 & $40 \%$ & 0 & $0 \%$ & 25 & $56 \%$ & 0 & $0 \%$ & 2.93 \\
\hline Clima organizacional & 8 & $18 \%$ & 20 & $44 \%$ & 7 & $16 \%$ & 10 & $22 \%$ & 0 & $0 \%$ & 3.57 \\
\hline \multicolumn{11}{|c|}{ Total } & 2.91 \\
\hline
\end{tabular}

Fuente: Autor

TABla VII. Estadísticos Para la dimensión TIC

\begin{tabular}{|c|c|c|c|c|c|c|c|c|c|c|c|}
\hline \multirow{2}{*}{ Indicadores } & \multicolumn{2}{|c|}{ TA } & \multicolumn{2}{|c|}{ MA } & \multicolumn{2}{|c|}{ NAND } & \multicolumn{2}{|c|}{ MD } & \multicolumn{2}{|c|}{ TD } & \multirow[t]{2}{*}{ Me } \\
\hline & $\mathbf{F a}$ & Fr & $\mathbf{F a}$ & Fr & $\mathbf{F a}$ & Fr & $\mathbf{F a}$ & Fr & $\mathbf{F a}$ & Fr & \\
\hline Uso para redes de conocimiento & 0 & $0 \%$ & 0 & $0 \%$ & 14 & $31 \%$ & 29 & $64 \%$ & 2 & $4 \%$ & 2.3 \\
\hline Herramienta para actividades de innovación & 0 & $0 \%$ & 18 & $40 \%$ & 0 & $0 \%$ & 27 & $56 \%$ & 0 & $0 \%$ & 2.8 \\
\hline \multicolumn{11}{|c|}{ Total } & 2.5 \\
\hline
\end{tabular}

Fuente: Autor 
En la Tabla VIII, se observan los resultados de los indicadores correspondientes a la variable estrategias de innovación, la cual obtuvo un valor para la media de 2.81, ubicándose en una categoría de moderado nivel.

Tabla VIII. Estadístico de LA VARIAble Estrategias de InNovación.

\begin{tabular}{|c|c|c|}
\hline Dimensiones & Media & Categoría \\
\hline Desarrollo de Actividades de I+D & 2.5 & Bajo nivel \\
\hline Capital humano & 3.33 & Moderado nivel \\
\hline Cultura organizacional & 2.91 & Moderado nivel \\
\hline $\begin{array}{l}\text { Tecnología de Información y } \\
\text { Comunicación }\end{array}$ & 2.53 & Bajo nivel \\
\hline Total & 2.81 & Moderado nivel \\
\hline
\end{tabular}

Fuente: Autor

La consideración en conjunto de las dimensiones correspondientes a la variable Estrategias de innovación se presenta en la Tabla VIII, donde se observa que la variable se ubica en la categoría de moderado nivel con una media aritmética de 2.81.

Estos resultados dejan entrever, de acuerdo con las respuestas de los individuos encuestados, que la variable Estrategias de innovación posee un moderado nivel en cuanto al capital humano y la cultura organizacional y un bajo nivel de desarrollo de actividades de I+D y de uso de las Tecnologías de Información y Comunicación. Esto indica que en los centros de investigación de las universidades públicas no se están diseñando estrategias efectivas de innovación que propicien el incremento tanto de la competitividad como de la rentabilidad de las organizaciones mediante el fomento de la innovación y la mejora continua de la calidad de los servicios y productos que se generan en este tipo de organizaciones.

Los resultados anteriormente descritos no son acordes con los planteamientos realizados por algunos autores que sugieren como estrategias de innovación las dimensiones analizadas, ya que éstas no se están desarrollando de manera eficaz, convirtiéndose en barreras que disminuyen las posibilidades de innovación de los centros de investigación objeto de estudio.

En cuanto al desarrollo de actividades de I+D, la referencia [8] expresa que estas actividades constituyen una estrategia fundamental de innovación y representan un mecanismo por excelencia para organizar y dirigir los procesos innovadores en la empresa. Por otra parte, señala [14] que si las organizaciones cuentan con un capital humano altamente calificado con competencias para desarrollar a cabo proyectos de innovación, entonces decidirá llevar a cabo procesos de innovación; también [7] sostiene que una estrategia debe basarse en el conocimiento del talento humano para mejorar el logro de los objetivos de la organización.
En referencia a la cultura innovadora, para que ésta exista, afirma [21], es necesario que se genere un clima organizacional que estimule la creatividad y se lideren procesos innovadores, y para ello se necesita el esfuerzo de todos los trabajadores dentro de la organización.

En cuanto a las TIC, [25] expresa que éstas desempeñan un papel funcional y de soporte a las estrategias competitivas de las organizaciones; sin embargo, en los centros de investigación no se están considerando como uno de los factores básicos de la innovación.

\section{Conclusiones y Recomendaciones}

Los resultados obtenidos en la investigación realizada dirigida a analizar las estrategias de innovación desarrolladas en los centros de investigación de las universidades públicas del Departamento de La Guajira, permiten señalar que existe una moderada presencia de estrategias de innovación en los centros de investigación de las universidades públicas del Departamento de La Guajira, siendo el aspecto más favorecido el capital humano existente y la cultura organizacional, mientras los aspectos menos favorecidos son la Tecnología de Información y Comunicación utilizada y el desarrollo de actividades de I+D.

Se pudo observar que los centros de investigación de las universidades públicas del Departamento de La Guajira cuentan con escaso personal con las competencias necesarias para el desarrollo de actividades de $\mathrm{I}+\mathrm{D}$, destinan poco presupuesto y los investigadores se sienten poco incentivados para el desarrollo de este tipo de actividades, pues se otorgan mínimamente estímulos al personal dedicado a la investigación por su aporte a la CTeI, así se aprovechan moderadamente los conocimientos del talento humano.

Del mismo modo se observa que de manera moderada se tiene una cultura en la organización que podría contribuir al fomento de la innovación de los investigadores.

Además, se promueve poco el uso de las TIC como herramienta para el desarrollo de actividades de innovación y para realizar contactos con redes de conocimiento, constituyéndose esto en una barrera de comunicación entre los grupos de investigación y con otras universidades.

Por lo anterior, se recomienda realizar un seguimiento continuo a los procesos desarrollados en los centros de investigación con el propósito de establecer estrategias de innovación.

Concretamente, se requieren acciones de la alta dirección que busquen fomentar el mejoramiento continuo de los servicios que presta. Es necesario potenciar las actividades ligadas a la $\mathrm{I}+\mathrm{D}+\mathrm{I}$ que sean generadoras de avances sociales y, que de esta forma, se conviertan en incremento de calidad de vida de la zona de influencia de las universidades. 
Se requiere llevar a cabo investigación aplicada mediante la generación de materiales y productos, cuyo objetivo principal sea resolver problemas de las empresas de la región.

Desde esa perspectiva, es necesario que se establezca un plan de capacitación para el fomento de la formación de investigadores con el fin de incrementar sus capacidades en CTI. Inicialmente, se debe realizar un análisis riguroso de los requerimientos de las áreas específicas para la formación de los grupos de investigación en I+D+I, así como establecer programas de incentivos para el fortalecimiento de la investigación y desarrollo de nuevos productos, procesos y servicios, ya sea generados por iniciativa propia o por la vinculación universidad-empresa.

Adicionalmente, es fundamental destinar recursos que permitan financiar de manera eficiente y efectiva las actividades de I+D en las líneas que trabajan cada uno de los grupos de investigación. Asimismo, establecer programas para la adquisición y uso de Tecnologías de Información y Comunicación (TIC) que sirvan como soporte a los grupos de investigación. De la misma manera, se debe propiciar un ambiente que fomente la creatividad, incorporando las actividades de innovación en los lineamientos estratégicos tales como misión, visión, valores, principios, buscando así facilitar los procesos de innovación en los centros de investigación de este tipo de instituciones.

\section{REFERENCIAS}

[1] M. Henao y J. Castro, Estados del arte de la investigación en educación y pedagogía en Colombia. Bogotá- Colombia: Icfes- Colciencias -Socolpe, 2000.

[2] C. Barrios, "Innovación y productividad científica en los institutos universitarios de tecnología de la Región Andina," Tesis de doctorado, Dept. Cien. Univ. Dr. Rafael Belloso Chacín, Maracaibo, Venezuela, 2013, pp. 122128.

[3] E. E. Bonilla Blanchar, "Cultura organizacional e innovación tecnológica en las instituciones públicas de educación superior, zona fronteriza colombo-venezolana (estado Zulia-Departamento de la Guajira)," Omnia, vol. 17, no. 3, 2011, pp. 86-98.

[4] J. W. Zartha Sossa, G. L. Orozco Mendoza, J. I. Vergara Sornoza y D. J. Martínez, "Diagnóstico de estrategia de innovación en grupos de investigación," J. Technol. Manag. Innov., vol. 6, no. 3, 2011, pp. 196-206.

[5] R. Abello y K. Pardo Sánchez, "Modelos de investigación y desarrollo en instituciones de educación superior en Colombia: El caso de la Universidad del Norte en la región Caribe de Colombia," Investig. Desarro., vol. 22, no. 2, 2014.

[6] E. Fernández, Estrategia de Innovación. Madrid: Ediciones Paraninfo, ISBN 13: 9788497324120 , mayo 2005, pp. $40-46$.
[7] Á. L. Arboniés, La disciplina de la innovación. Argentina: Díaz de Santos, 2009, pp. 122-125.

[8] J. Vega Jurado, A. Gutiérrez Gracia y F. Jiménez Sáez, "Estrategias de innovación en la industria manufacturera española: sus determinantes y efectos sobre el desempeño innovador," Tesis de doctorado, Dept. Proy. Ing., Univ. Politéc.Valencia, Valencia, España, 2008.

[9] OCDE y Eurostat, Manual de Oslo. Guía para la recogida e interpretación de datos sobre innovación, 3rd ed. París: OCDE, 2005, pp. 106-107.

[10] OCDE, Propuesta de Norma Práctica para Encuestas de Investigación y Desarrollo Experimental. España, 2002, pp. 16-18.

[11] M. L. Medina, M. G. Medina y L. A. Merino, "La investigación científica como misión académica de los hospitales públicos universitarios," Rev. Cuba. Salud Pública, vol. 41, no. 1, 2015, pp. 139-146.

[12] R. Loubet-Orozco y A. Morales-Parra, "Formación de capital humano para el crecimiento económico en Sinaloa," Ra Ximhai, vol. 11, no. 3, 2015, pp. 41-55.

[13] F. E. Hernández Silva y Y. Martí Lahera, "Conocimiento organizacional: la gestión de los recursos y el capital humano," ACIMED, vol. 14, no. 1, 2007.

[14] L. Á. Guerras Martín y J. E. Navas López, La dirección estratégica de la empresa: teoría y aplicaciones, 3rd ed. Pamplona: S.L. Civitas Ediciones, 2007, pp. 35-42.

[15] M. J. Sánchez Bueno, El proceso innovador y tecnológico: estrategias y apoyo público, 1st ed. España: Netbiblo, 2008, pp. $17-20$.

[16] A. M. Segredo Pérez, A. J. García Milian, P. López Puig, P. León Cabrera, y I. Perdomo Victoria, "Enfoque sistémico del clima organizacional y su aplicación en salud pública," Rev. Cuba. Salud Pública, vol. 41, no. 1, 2015.

[17] K. Davis y J. Newstrom, Comportamiento humano en el trabajo, 11th ed. México: Mc Graw Hill, 2003, pp. 11-29.

[18] I. Chiavenato, Administración de Recursos Humanos, 8th ed. México: McGraw-Hill, 2007, pp. 93-111.

[19] S. P. Robbins y T. A. Judge, Comportamiento organizacional, 13th ed. México: Pearson, 2009, pp. 172-175.

[20] D. Minsal Pérez y Y. Pérez Rodríguez, "Hacia una nueva cultura organizacional: la cultura del conocimiento," ACIMED, vol. 16, no. 3, 2007.

[21] D. Touche and Tohmatsu, "Fostering an innovative culture," 2004, pp. 12-20. [En línea]. Disponible en: http:// wwwdeloite.com/growler.

[22] A. Lagunes-Domínguez, C. A. Torres-Gastelú, M. A. Flores-García y A. Rodríguez-Figueroa, "Comparativo del uso de Tecnologías de la Información y Comunicación (TIC) por profesores de dos universidades públicas de México," Form. Univ., vol. 8, no. 2, 2015, pp. 11-18. DOI:http://dx.doi.org/10.4067/S071850062015000200003

[23] L. González P, S. Arango, C. Vásquez y J. Ospina, "Campo de investigación en tecnologías de información y comunicación: estrategia de gobernanza en la Universidad de Medellín," Ingeniare. Rev. Chil. Ing., vol. 23, no. 2, 2015, pp. 301-311.

[24] M. C. Arbeláez Gómez, "Las tecnologías de la información y la comunicación (TIC) un instrumento para la investigación," Investig. Andin., vol. 16, no. 29, 2014, pp. 997-1000.

[25] Naciones Unidas para el Desarrollo, "Las nuevas tecnologías, ¿un salto al futuro?," Desarrollo Humano en Chile, Santiago de Chile, 2006, pp. 20-31. 